\title{
Multiple CMS-restorer gene polymorphism in gynodioecious Plantago coronopus
}

\author{
JMM van Damme ${ }^{1}$, MPJ Hundscheid ${ }^{1}$, S Ivanovic $^{1}$ and HP Koelewijn ${ }^{2}$ \\ ${ }^{1}$ Netherlands Institute of Ecology, Center for Terrestrial Ecology, PO Box 40, 6666 ZG Heteren, The Netherlands; ${ }^{2}$ Alterra, Center for \\ Ecosystem Studies, PO Box 47, 6700 AA Wageningen, The Netherlands
}

\begin{abstract}
The mode of inheritance of the male sterility trait is crucial for understanding the evolutionary dynamics of the sexual system gynodioecy, which is the co-occurrence of female and hermaphrodite plants in natural populations. Both cytoplasmic (CMS) and nuclear (restorer) genes are known to be involved. Theoretical models usually assume a limited number of CMS genes with each a single restorer gene, while reality is more complex. In this study, it is shown that in the gynodioecious species Plantago coronopus two new CMS-restorer polymorphisms exist in addition to the two that were already
\end{abstract}

known, which means four CMS-restorer systems at the species level. Furthermore, three CMS types were shown to co-occur within a single population. All new CMS types showed a multilocus system for male fertility restoration, in which both recessive and dominant restorer alleles occur. Our finding of more than two co-occurring CMS-restorer systems each with multiple restorer genes raises the question how this complex of male sterility systems is maintained in natural populations. Heredity (2004) 93, 175-181, advance online publication, 12 May 2004; doi:10.1038/sj.hdy.6800490

Keywords: gynodioecy; restorer genes; reciprocal crosses; genetics; cytoplasm; male sterility

\section{Introduction}

The maintenance and evolution of gynodioecy, which is the coexistence of female and hermaphroditic plants in natural populations, has received attention since Lewis (1941) recognized the fitness problem associated with this sexual system. Because female plants do not pass on genes to the next generation through pollen, they should compensate this fitness disadvantage somehow via the female pathway of gene transmission by a higher lifetime seed output. Lewis already distinguished the fitness consequences of two possible modes of inheritance of the male sterility trait: by cytoplasmic genes (CMS) and by nuclear genes. The latter are usually referred to as restorer genes. Later authors (Frank, 1989; Gouyon et al, 1991; Maurice et al, 1994; Schultz, 1994; McCauley and Taylor, 1997) have expanded Lewis' theory to include simultaneous CMS-restorer gene inheritance of male sterility, which was necessary because of the empirical evidence of this mode of inheritance in gynodioecious species (Kheyr-Pour, 1981; Van Damme and Van Delden, 1982; Belhassen et al, 1991; Cuguen et al, 1994; Koelewijn and Van Damme, 1995a; Charlesworth and Laporte, 1998).

With the exception of Frank (1989, see discussion), these models typically have studied either the combination of two CMS-restorer gene systems, or of one CMSrestorer gene system and one male fertile cytoplasm. The maintenance of joint CMS-restorer gene polymorphism in these (relatively simple) genetic models is not yet well

Correspondence: JMM van Damme, Netherlands Institute of Ecology, Center for Terrestrial Ecology, PO Box 40, 6666 ZG Heteren, The Netherlands. E-mail: j.vandamme@nioo.knaw.nl

Received 21 April 2003; revised 10 October 2003; accepted 30 November 2003; published online 12 May 2004 understood since the conditions for maintenance have appeared either to be rather stringent (Gouyon et al, 1991) or to depend strongly on continuous reintroduction of CMS and restorer genes from the metapopulation to counteract local extinction of male sterility (Frank, 1989; McCauley and Taylor, 1997).

Natural CMS-restorer gene polymorphisms are even more complex than these existing models. First, in contrast to the usual assumption of a single restorer gene for a CMS type, in well-studied gynodioecious species multiple restorer genes, both recessive and dominant, have been found for each CMS type (Van Damme, 1983; Koelewijn and Van Damme, 1995b; Charlesworth and Laporte, 1998). Second, the number of CMS-restorer gene systems simultaneously engaged in natural populations can exceed the one or two, usually assumed in the models.

On theoretical grounds, a relatively low number of CMS types in gynodioecious species would be expected. The mechanistic cause of CMS is generally believed to be located in the mitochondrial DNA (mt-DNA) (SaumitouLaprade et al, 1994; Schnable and Wise, 1998). Mutations in the mt-DNA have two disadvantages compared to a mutation in a nuclear gene. Firstly, it is immediately exposed to competition with the other mt-DNA molecules in the cell. The mutation has to become fixed at the cell level either by chance or by a competition advantage like replication speed. Secondly, it needs to be at least neutral with respect to cell survival. In contrast, a recessive nuclear mutation can be hidden from selection in the heterozygous state, because of diploidy of higher plants.

Empirical studies of the number of co-occurring CMS types and their mode of fertility restoration in gynodioecious plant species are scarce. Genetic evidence for the presence of more than two CMS systems at the species 
level exists only in gynodioecious Plantago lanceolata (De Haan et al, 1997) and in the crop species Zea mays (Beckett, 1971). In several other gynodioecious species, considerable mt-DNA variation within populations has been found, but genetic evidence by differences in reciprocal crosses for their CMS status is mostly lacking (Belhassen et al, 1993; Cuguen et al, 1994; Ronfort et al, 1995).

Here we report on the extent of CMS polymorphism and the complexity of their restorer genetics in the gynodioecious species Plantago coronopus. In this species, two CMS systems have been characterized each with a complex set of restorer genes (Koelewijn and Van Damme, 1995a, b). A crossing program was set up with a number of candidate field-collected plants in order to answer the following specific questions:

- Do more than two CMS systems occur in gynodioecious $P$. coronopus?

- Do the new CMS systems have a single or multiple restorer genes?

- Can individual populations be polymorphic for more than two CMS types?

\section{Materials and methods}

\section{Plant material}

$P$. coronopus is a wind pollinated, self-compatible annual or short-lived perennial, growing mainly in dune grasslands along the coast. Each flower has five ovules, and four anthers that each contain approximately 13000 pollen grains (HP Koelewijn, unpublished results). The plants used in this study came from three sites in the Netherlands - Kwade Hoek (kh), Oostvoornse Meer (om) and Lorentzsluizen (lora) - and from Achill Island, Ireland (ei). Collection years were 1989 for the ei4ms plant and 2000 for the lora4 plant. The $\mathrm{kh}$ and om plants were mostly taken from an earlier study (Koelewijn and Van Damme, 1995b).

\section{Growing conditions}

A maximum of 50 seeds were germinated in Petri dishes of $9 \mathrm{~cm}$ diameter on $0.7 \%$ agar-containing medium. Crosses were sown in six batches from September 2000 until September 2002. Germination rates among crosses were on average 0.86 . Preliminary analysis showed a significant effect on germination rate of sowing date $(\mathrm{F}(5,95)=4.69, P<0.001$; range $0.82-0.96)$, but no effects of the female parent, the male parent and of CMS type (not shown). Seedlings were planted out in a greenhouse (temp. d/n $21 / 16^{\circ} \mathrm{C}$, day length $16 \mathrm{~h}$, light level minimal $225 \mu \mathrm{mol} / \mathrm{ms}$ PAR) 5-10 days after germination, and kept in 0.31 pots filled with potting soil. In total, 5500 plants were grown from 96 crosses, that is, on average 57.3 progeny per cross.

\section{Crossing procedure}

Potential cytoplasmic types were tested in reciprocal crosses with plants of known CMS type as tester plants: CMS1 and CMS2 (Koelewijn and Van Damme, 1995a). Differences between reciprocals in segregation of male sterile plants (ms) and non-male sterile plants (non-ms) serve as evidence for a separate CMS status of the tested plant, because they reveal that different nuclear loci are involved in the restoration of the novel and the known cytoplasmic type. The optimal genetic constitution for a tester plant is to have a CSM type that is rarely restored, because that maximizes the probability of sex segregation in the cross it mothers, and the likelihood of detecting differences depends on the occurrence of such sex segregation.

The potential CMS types tested this way were represented by the four field-collected plants (ei4ms, lora4, kh2010 and kh120) and the families derived from these plants (Table 1).

In the crosses, we took advantage of the protogynous flowering habit. Inflorescences smaller than $4 \mathrm{~cm}$ usually flower fully female before male flowering starts. When inflorescences were longer than $4 \mathrm{~cm}$, pollination was carried out before female flowering had reached the top, and this underdeveloped top was cutoff to avoid later unwanted pollination. Scissors were cleaned between crosses. Pollen was collected in a $1.5 \mathrm{ml}$ eppendorf tube by inserting a male flowering inflorescence and shaking it gently. Self-fertilization was subsequently achieved by moving the inflorescence up and down several times in the eppendorf tube. For crosses, a virgin female flowering inflorescence was dipped in a pollen-containing eppendorf tube, and gently rolled around. After pollination, the style was usually covered with pollen. The inflorescence was labeled with a marked paper-cloth label and left on the plant for about a month until the seeds were ripe. Pollinated plants were carefully separated in space so that inflorescences did not overlap for at least $24 \mathrm{~h}$, which is the time within which fertilization takes place.

Offspring of crosses were sexed (ms versus non-ms) by eye at the age of 8-10 weeks when they had at least five male (-sterile) flowering spikes.

\section{Data analysis}

Segregation data were analyzed with $\chi^{2}$ tests for heterogeneity for separate reciprocal crosses, as well as

Table 1 Crossing history of plant families used in this study (two sets of families with known CMS types CMS1 and CMS2, and families based on four plants, ei4, lora4, kh2010 and kh120, that represent potential CMS sources)

\begin{tabular}{|c|c|c|c|}
\hline Family & Cross & Family & Cross \\
\hline \multicolumn{2}{|c|}{ CMS1 tester families } & \multicolumn{2}{|c|}{ CMS2 tester families } \\
\hline G141 & kh51 × w95 & G142 & kh56ms $\times$ om 2 \\
\hline H1 & om2-s1 & H12 & kh41 selfing \\
\hline H77 & om $2 \times$ om 8 & H18 & $\mathrm{om} 46 \mathrm{~ms} \times \mathrm{om} 8$ \\
\hline K466 & om90-s2 & H19 & om $46 \mathrm{~ms} \times$ om 17 \\
\hline K468 & om $90-\mathrm{s} 1 \times(\mathrm{kh} 56 \times \mathrm{kh} 51)$ & $\mathrm{H} 21$ & om $60 \mathrm{~ms} \times \mathrm{om} 8$ \\
\hline
\end{tabular}

Crosses derived from field-collected plants

G140 lora4 $\times$ G141-100

K165 kh120-s1-2ms $\times$ kh120-s1-4

K183 kh120-s1-8ms $\times$ kh120-s1-20

K465 kh2010-s1

K467 ei4ms $\times$ kh2010

In the crosses, the female parent is mentioned first. The parents used were hermaphrodite unless they have ms for male sterile in their name. The name components s1 and s2 are first and second generation of self-fertilization, respectively. Families with $\mathrm{H}$ in the name refer to crosses published in Koelewijn and Van Damme (1995a, b). 
for groups of individual crosses that shared the same optimal ratio. The best-fitting Mendelian ratio for each cross was determined by testing all possible ratios by $\chi^{2}$, assuming a two-locus system of fertility restoration. When there was only one degree of freedom, the correction for continuity was used (Snedecor and Cochran, 1977).

\section{Results}

\section{Cytoplasmic differences}

Preliminary research suggested that at least three $P$. coronopus plants carried a male sterile cytoplasm different from the previously known CMS types, CMS1 and CMS2 (Koelewijn and Van Damme, 1995a). These plants were subsequently compared with CMS1 and CMS2 and with each other in a series of reciprocal crosses. Of these plants, lora4 showed a clear significant reciprocal difference when crossed with a single CMS1 tester, and its F1 progeny (for parentage in this and following cases, see Table 1), crossed to one of four CMS2 testers with different nuclear genetic background, showed significant differences in five out of six cases (Table 2), which supports a distinct cytoplasmic status of plant lora4. Both the CMS1 and CMS2 female parents as well as the parents with lora4 cytoplasm segregated male steriles.

Reciprocal differences in crosses with CMS1 and CMS2 also showed up for plant ei4ms. Because ei4ms was a male sterile plant, four hermaphroditic F1 full sibs with ei4ms cytoplasm were used for crosses with three CMS1 testers and with four CMS2 testers of different nuclear genetic background in incomplete diallele designs. In tests with CMS1 three out of eight reciprocal crosses showed significant differences, and with CMS2 three out of 11 cases. Remarkably, while cytoplasmically distinct from CMS1 and CMS2, plants with ei4ms cytoplasm hardly segregated male steriles. This was also the case when ei4ms and lora4 cytoplasmic types were tested against each other: five out of seven crosses showed significantly different sex segregation among reciprocals with the lora4 cytoplasmic type segregating and the ei4ms type hardly or not (Table 3 ).

Thirdly, plant kh2010 appeared different from CMS1 in one out of two crosses, and different from CMS2 in two out of four crosses, confirming its status as a distinct cytoplasmic type relative to CMS1 and CMS2 (Table 4). Plant kh2010 was also used as the male parent in a backcrossing program with cytoplasmic type ei4ms. Here ei4ms cytoplasm appeared very well capable of

Table 2 Reciprocal crosses between two potential cytoplasmic sources (represented by plants lora4 and ei4ms) with known tester plants from CMS1 and CMS2

\begin{tabular}{|c|c|c|c|c|c|c|c|c|c|c|c|c|c|}
\hline \multirow[t]{2}{*}{ Mother } & \multirow[t]{2}{*}{ Father } & \multirow[t]{2}{*}{ Field source mother } & \multirow[t]{2}{*}{$m s$} & \multirow[t]{2}{*}{ non-ms } & \multirow[t]{2}{*}{ Family } & \multicolumn{2}{|c|}{ Reciprocal cross } & \multicolumn{2}{|c|}{ Heterogeneity } & \multirow[t]{2}{*}{ Ratio } & \multirow[t]{2}{*}{$\chi^{2}(1)$} & \multirow[t]{2}{*}{ Ratio } & \multirow[t]{2}{*}{$\chi^{2}(1)$} \\
\hline & & & & & & $m s$ & non-ms & $\chi^{2}(1)$ & $\mathrm{P}$ & & & & \\
\hline Cytoplasmic type & & & \multicolumn{2}{|c|}{ CMS1 } & & \multicolumn{2}{|c|}{ lora4 } & & & CMS1 & & lora4 & \\
\hline G141-1 & lora4 & Kh51 & 25 & 59 & & 6 & 74 & 13.31 & 0.000 & $1: 3$ & $0.78 \mathrm{NS}$ & $1: 7$ & $1.40 \mathrm{NS}$ \\
\hline Cytoplasmic type & & & \multicolumn{2}{|c|}{ CMS1 } & & \multicolumn{2}{|l|}{ lora4 } & & & \multicolumn{2}{|l|}{ CMS1 } & lora4 & \\
\hline G142-1 & G140-1 & Kh56 & 1 & 53 & K633 & 15 & 63 & 9.35 & 0.002 & - & & $1: 3$ & $1.09 \mathrm{NS}$ \\
\hline G142-1 & G140-3 & Kh56 & 0 & 107 & K635 & 23 & 109 & 20.85 & 0.000 & $0: 1$ & & $1: 7$ & $2.49 \mathrm{NS}$ \\
\hline H18-100 & G140-6 & om46 & 10 & 54 & & 1 & 74 & 9.65 & 0.002 & $1: 7$ & $0.32 \mathrm{NS}$ & - & \\
\hline H19-100 & G140-8 & om46 & 1 & 56 & & 0 & 141 & 2.25 & 0.134 & - & & $0: 1$ & \\
\hline H21-100 & G140-9 & om60 & 8 & 63 & & 3 & 94 & 4.40 & 0.036 & $1: 7$ & $0.02 \mathrm{NS}$ & $1: 15$ & $1.16 \mathrm{NS}$ \\
\hline H18-100 & G140-10 & om46 & 6 & 72 & & 0 & 57 & 5.02 & 0.025 & $1: 7$ & $1.24 \mathrm{NS}$ & $0: 1$ & \\
\hline Cytoplasmic type & & & \multicolumn{2}{|c|}{ CMS1 } & & \multicolumn{2}{|c|}{ ei4ms } & & & \multicolumn{2}{|l|}{ CMS1 } & ei4ms & \\
\hline H1-100 & K467-6 & om2 & 12 & 50 & K680 & 0 & 36 & 8.40 & 0.004 & $1: 3$ & $0.77 \mathrm{NS}$ & $0: 1$ & \\
\hline K466-2 & K467-6 & om90 & 9 & 86 & & 0 & 100 & 10.00 & 0.002 & $1: 7$ & $0.54 \mathrm{NS}$ & $0: 1$ & \\
\hline K468-2 & K467-6 & om90 & 0 & 79 & & 0 & 80 & & & $0: 1$ & & $0: 1$ & \\
\hline H77-16b & K467-85 & om2 & 2 & 52 & & 0 & 64 & 2.74 & 0.098 & $1: 7$ & $3.06 \mathrm{NS}$ & $0: 1$ & \\
\hline K466-2 & K467-85 & om90 & 1 & 13 & & 0 & 40 & 2.42 & 0.120 & $1: 3$ & $1.52 \mathrm{NS}$ & $0: 1$ & \\
\hline K468-2 & K467-85 & om90 & 0 & 50 & & 0 & 43 & & & $0: 1$ & & $0: 1$ & \\
\hline H1-100 & K467-77 & om2 & 19 & 46 & & 0 & 60 & 20.81 & 0.000 & $1: 3$ & $0.42 \mathrm{NS}$ & $0: 1$ & \\
\hline K468-2 & K467-77 & om90 & 0 & 41 & & 0 & 38 & & & $0: 1$ & & $0: 1$ & \\
\hline \multicolumn{3}{|l|}{ Cytoplasmic type } & \multicolumn{2}{|c|}{ CMS2 } & & \multicolumn{2}{|c|}{ ei4ms } & & & CMS2 & & eitms & \\
\hline H12-10 & K467-6 & Kh41 & 4 & 28 & & 4 & 91 & 2.35 & 0.125 & $1: 15$ & $1.20 \mathrm{NS}$ & $1: 15$ & $0.37 \mathrm{NS}$ \\
\hline H12-27 & K467-6 & Kh41 & 0 & 42 & & 0 & 23 & & & $0: 1$ & & $0: 1$ & \\
\hline H18-100 & K467-6 & om46 & 10 & 39 & & 0 & 34 & 8.27 & 0.004 & $1: 3$ & $0.33 \mathrm{NS}$ & $0: 1$ & \\
\hline G142-1 & K467-6 & Kh56 & 3 & 28 & & 2 & 43 & 0.90 & 0.342 & $1: 7$ & $0.04 \mathrm{NS}$ & $1: 7$ & $1.98 \mathrm{NS}$ \\
\hline H12-17 & K467-77 & Kh41 & 0 & 50 & & 0 & 49 & & & $0: 1$ & & $0: 1$ & \\
\hline H12-27 & K467-77 & Kh41 & 0 & 49 & & 0 & 50 & & & $0: 1$ & & $0: 1$ & \\
\hline H18-100 & K467-77 & om46 & 11 & 49 & K684 & 0 & 17 & 4.39 & 0.036 & $1: 3$ & $1.09 \mathrm{NS}$ & $0: 1$ & \\
\hline G142-1 & K467-77 & Kh56 & 0 & 57 & & 0 & 15 & & & $0: 1$ & & $0: 1$ & \\
\hline H12-17 & K467-85 & Kh41 & 0 & 17 & & 0 & 39 & & & $0: 1$ & & $0: 1$ & \\
\hline G142-1 & K467-85 & Kh56 & 5 & 62 & & 0 & 49 & 4.29 & 0.038 & $1: 7$ & $1.13 \mathrm{NS}$ & $0: 1$ & \\
\hline H12-27 & K467-41 & Kh41 & 0 & 48 & & 0 & 49 & & & $0: 1$ & & $0: 1$ & \\
\hline
\end{tabular}

In a single row, ms versus non-ms segregations of reciprocal crosses are given, as well as the $\chi^{2}$ for heterogeneity among reciprocals and for the optimal ratio for each cross separately when applicable. Significant probability $(P)$ values are indicated in boldface. The family numbers provide the link between these crosses and the next generation shown in Table 3 . 
Table 3 Reciprocal crosses between the two potential cytoplasmic types, represented by plants ei4ms and lora 4

\begin{tabular}{|c|c|c|c|c|c|c|c|c|c|c|c|}
\hline \multirow[t]{2}{*}{ Mother } & \multirow[t]{2}{*}{ Father } & \multirow[t]{2}{*}{$m s$} & \multirow[t]{2}{*}{ non-ms } & \multicolumn{2}{|c|}{ Reciprocal cross } & \multicolumn{2}{|c|}{ Heterogeneity } & \multirow[t]{2}{*}{ Ratio } & \multirow[t]{2}{*}{$\chi^{2}(1)$} & \multirow[t]{2}{*}{ Ratio } & \multirow[t]{2}{*}{$\chi^{2}(1)$} \\
\hline & & & & $m s$ & non-ms & $\chi^{2}(1)$ & $\mathrm{P}$ & & & & \\
\hline \multicolumn{2}{|c|}{ Cytoplasmic type } & \multicolumn{2}{|c|}{ eitms } & \multicolumn{2}{|c|}{ lora4 } & & & eitms & & lora4 & \\
\hline K467-85 & G140-3 & 0 & 59 & 3 & 25 & 5.88 & 0.015 & $0: 1$ & & $1: 7$ & $0.00 \mathrm{NS}$ \\
\hline K467-77 & G140-6 & 0 & 46 & 10 & 36 & 11.33 & 0.001 & $0: 1$ & & $1: 3$ & $0.12 \mathrm{NS}$ \\
\hline K467-6 & G140-8 & 1 & 46 & 0 & 47 & & & - & & $0: 1$ & \\
\hline K684-202 & K633-125 & 0 & 48 & 17 & 30 & 21.20 & 0.000 & $0: 1$ & & $1: 3$ & $2.56 \mathrm{NS}$ \\
\hline K684-205 & K635-140 & 0 & 55 & 12 & 39 & 14.62 & 0.000 & $0: 1$ & & $1: 3$ & $0.01 \mathrm{NS}$ \\
\hline K680-201 & K633-128 & 3 & 37 & 0 & 41 & 3.52 & 0.061 & $1: 7$ & $0.51 \mathrm{NS}$ & $0: 1$ & \\
\hline K680-202 & K635-131 & 0 & 20 & 13 & 29 & 8.33 & 0.004 & $0: 1$ & & $1: 3$ & $0.51 \mathrm{NS}$ \\
\hline
\end{tabular}

Families K633, K635, K680 and K684 are from crosses presented in Table 2. For legend, see Table 2.

Table 4 Crosses with cytoplasmic source kh2010 from population de Kwade Hoek

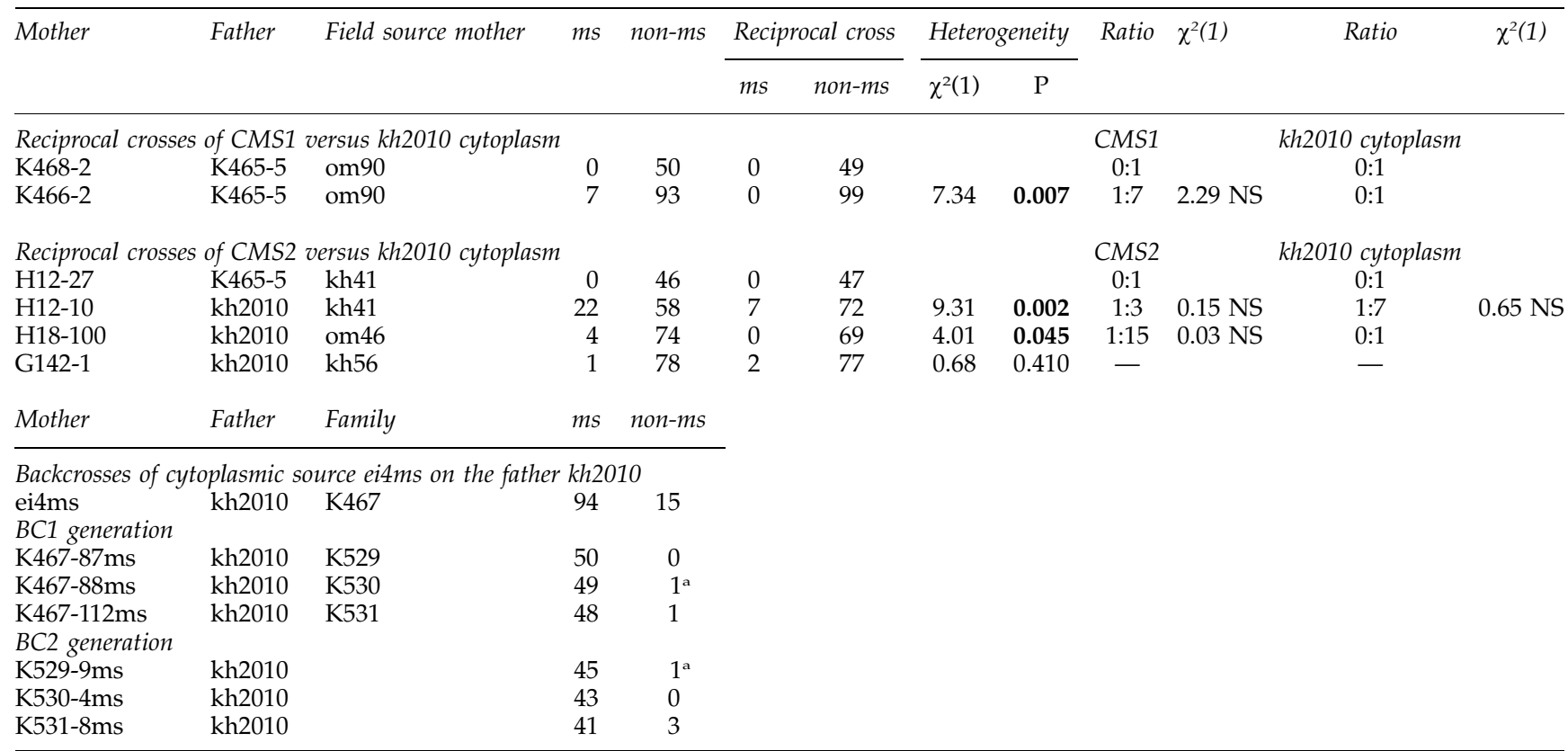

For legend, see Table 2.

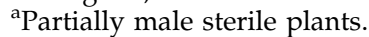

segregating male sterile plants. Backcrossing three different ms progeny from the ei $4 \mathrm{~ms} \times \mathrm{kh} 2010$ cross on the father for two consecutive generations gave only ms progeny or nearly so. The single non-ms plants in two of the crosses were partially male sterile (Table 4). In two of the three backcross lines, plant kh2010 acts as a maintainer of male sterility for cytoplasmic type of plant ei4ms, which makes it likely that plant kh2010 is cytoplasmically distinct from ei4ms cytoplasm as well.

Finally, we report here a re-examination of the case of plant kh120, which had previously been classified as CMS1 by Koelewijn and Van Damme (1995a) because it showed differences with CMS2 in three out of four crosses, but not with CMS1 in three crosses (Table 5). Here we report of nine additional reciprocal crosses between F1 seed-descendants of kh120 and two CMS1 testers of different nuclear genetic background: five of the nine crosses clearly showed significant differences, thereby establishing that kh120 also represents a distinct cytoplasmic type from CMS1 as well as CMS2.

\section{Restorer status of CMS types}

When a single nuclear gene is involved in fertility restoration, one expects segregation ratios in units of $1 / 4$, like 1:1 and 1:3. Similarly, one expects ratios in units of $1 / 16$ for two-locus systems, and so forth. Sex ratios were fitted for all crosses in Tables 2, 3 and 4 that showed a fitted ratio of at least $1: 15$, corresponding to maximally two restorer loci. Crosses with the same individually fitted ratio were then grouped per CMS type. Summed over CMS types, crosses with the same sex ratio all appeared to be homogeneous, and each group totally fitted the ratio of that group except for the 1:7 ratio (Table 6). None of the possible two-locus ratios fitted the latter group. The nearest fitting ratio appeared to be 3:29 $\left(\chi^{2}=0.20, \mathrm{df}=1, \mathrm{NS}\right)$, suggesting at least three restorer loci to be involved in this crossing group.

Cytoplasmic types differed in the fraction of test crosses that segregated for male sterility (segregating versus nonsegregating, $\chi^{2}=36.21, \mathrm{df}=5, P<0.0001$; see Table 6). For example, kh120 cytoplasm segregated male 
Table 5 Reciprocal crosses of cytoplasmic source kh120 from population de Kwade Hoek

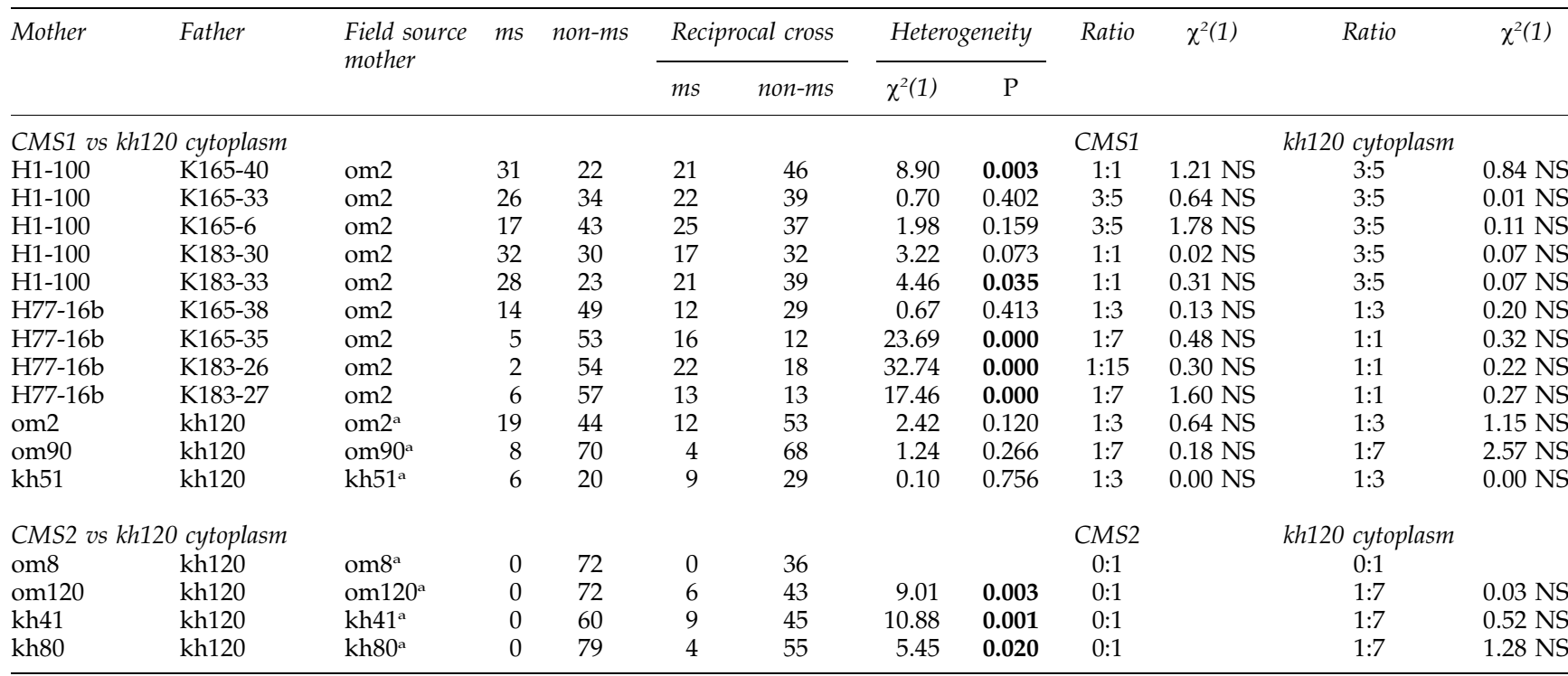

For legend, see Table 2.

${ }^{a}$ Results for these reciprocal crosses were taken from Koelewijn and van Damme (1995a, Table 8).

Table 6 Summary of the sex ratio results obtained in Tables $2-4$

\begin{tabular}{|c|c|c|c|c|c|c|c|c|}
\hline \multirow[t]{2}{*}{ CMS type } & \multicolumn{6}{|c|}{ Ratio } & \multirow[t]{2}{*}{ Not fitted } & \multirow[t]{2}{*}{ Total } \\
\hline & $1: 15$ & $1: 7$ & $1: 3$ & $3: 5$ & $1: 1$ & $0: 1$ & & \\
\hline CMS1 & 1 & 6 & 7 & 2 & 3 & 4 & 0 & 23 \\
\hline CMS2 & 2 & 5 & 3 & 0 & 0 & 12 & 3 & 25 \\
\hline CMSei & $\overline{1}$ & 2 & 0 & 0 & 0 & 22 & 1 & 26 \\
\hline CMSlora & 1 & 3 & 5 & 0 & 0 & 4 & 1 & 14 \\
\hline kh2010 cytoplasm & 0 & 1 & 0 & 0 & 0 & 4 & 1 & 6 \\
\hline kh120 cytoplasm & 0 & 4 & 3 & 5 & 3 & 1 & 0 & 16 \\
\hline Total & 5 & 21 & 18 & 7 & 6 & 47 & 6 & 110 \\
\hline$\chi^{2}$ heterogeneity & 5.1 & 23.10 & 16.15 & 4.11 & 0.85 & & & \\
\hline Degrees of freedom & 4 & 20 & 17 & 6 & 5 & & & \\
\hline$P$ & 0.28 & 0.28 & 0.51 & 0.66 & 0.97 & & & \\
\hline Total ms & 17 & 136 & 239 & 149 & 148 & & & \\
\hline Total non-ms & 341 & 1261 & 735 & 270 & 118 & & & \\
\hline$\gamma^{2}$ ratio & 1.10 & 9.50 & 1.30 & 0.60 & 2.00 & & & \\
\hline Degrees of freedom & 1 & 1 & 1 & 1 & 1 & & & \\
\hline$P$ & 0.29 & 0.002 & 0.77 & 0.44 & 0.15 & & & \\
\hline
\end{tabular}

Indicated are the numbers of crosses with a particular optimal ratio, sorted by CMS type, the heterogeneity $\chi^{2}$ for all crosses with the same optimal ratio (using the corresponding data from Tables $2-4$, not shown here), and the resulting total ms:non-ms numbers and the ratio $\chi^{2}$ on the total assuming the original optimal ratio. Significant probability $(P)$ values are indicated in boldface. For further explanation, see text.

steriles in all except one cross, whereas at the other extreme ei4ms cytoplasm segregated only male steriles in crosses with plant kh2010. Such differences suggest differences in restorer status among the CMS types as represented by the field-collected sources. Remarkably, the test plants used for CMS1, CMS2 and lora4 cytoplasm, coming from the Netherlands, all were (nearly) fully restored for ei4ms cytoplasm from Ireland.

The ratios 1:3 and 1:1 can be explained by a single locus with dominance of the restorer allele (R) or, alternatively formulated, recessiveness of the male sterility allele. Ratios 1:7 and 1:15 require two loci with dominance of $\mathrm{R}$ and independent restorer action. The situation for the 3:5 ratio is more complicated. The simplest model is a combination of two restorer loci, one with recessive ( $r$ ) and the other with dominant (R) restorer action (cf Lewis and Crowe, 1956), but then one parental genotype $(\mathrm{r}+++)$ has male sterile phenotype in its own CMS background, but is hermaphroditic in the other CMS background, which is possible in a cross between different CMS types.

Remarkably, all 3:5 and 1:1 crosses are confined to the crosses between a single CMS1 tester, plant H1-100, and plants representing kh120 cytoplasm (Table 6). Since, in 
this group, reciprocal crosses occur with a 3:5 ratio on either side, both CMS1 and kh120 cytoplasm may have a combination of a recessive and dominant restorer locus, and these two systems would then be at least partially independent of each other. Furthermore, since 1:7 ratios also occur in crosses with CMS1 and kh120 cytoplasm, it is likely that at least three restorer loci determine male sterility in both CMS types. For CMS1, this is in agreement with the earlier finding of Koelewijn and Van Damme (1995b).

\section{Discussion}

For the first time, we have presented conclusive evidence, based on reciprocal crosses, for the existence of four different CMS systems in a gynodioecious species, P. coronopus. Both lora4 and ei4ms cytoplasms were shown to differ from CMS1 and CMS2 and from each other. In addition, they are capable of segregating male steriles. We will designate lora 4 cytoplasm as CMS3 and ei4ms cytoplasm as CMS4. Cytoplasm kh2010 was shown to be genetically distinct from CMS1, CMS2 and CMS4, but has not been tested against CMS3. The presence of cytoplasm kh2010 does however show that in the population of Kwade Hoek three different cytoplasmic types co-occur, because CMS1 and CMS2 were shown to be present in that same population by Koelewijn and Van Damme (1995a). A similar situation has been found by de Haan et al (1997) in P. lanceolata.

The evidence from reciprocal crosses can be considered as conclusive because other causes for reciprocal differences can be ruled out including (1) linkage of a nuclear sex locus with a self-incompatibility locus ( $P$. coronopus is self-compatible) and (2) early acting differential selection on male sterility is very unlikely (the overall germination percentage is above $85 \%$, see Materials and methods), (3) the evidence in most cases is based on multiple fathers with different nuclear genetic backgrounds, which is a safeguard against misinterpretations due to rare exceptional results and also increases the power to detect differences.

Four CMS systems have also been claimed for P. lanceolata by de Haan et al (1997), but they used the sex type partial male sterility (PMS) in their analysis, which is known to be under strong environmental control as de Haan et al (1997) recognize themselves. Re-analysis of their data with the same sex criterion as in the present paper (ms versus non-ms) leads to evidence for three CMS types instead of four (mt types A vs $(B, C)$ versus (D, F); see de Haan et al, 1997). Kheyr-Pour (1981) has claimed a wide nuclear-cytoplasmic polymorphism in gynodioecious Origanum vulgare, which, however, judged by the criterion of reciprocal crosses with significant differences, shows evidence for only two CMS systems in this species. In wild Beta vulgaris, three $\mathrm{mt}$ types have been found to be associated with the occurrence of male sterility (Cuguen et al, 1994), but only two of them have been tested against each other in reciprocal crosses (Laporte et al, 1998). Evidence by reciprocal differences for two CMS types has also been found in Thymus vulgaris (Belhassen et al, 1991) and Silene vulgaris (Charlesworth and Laporte, 1998).

In the crop species Z. mays, only three CMS types ( $T, S$ and $C$ ) have been recognized in spite of tens of years of explicit research to find CMS variation (Duvick, 1965;
Beckett, 1971; Fauron and Casper, 1994; Fauron et al, 1995) after a serious crop failure in 1970 because of the susceptibility of CMS-T to the pathogen Helminthosporium maydis (Ullstup, 1972). In addition, two different so-called normal (N) cytoplasmic types are distinguished (Fauron and Casper, 1994). Male sterility is known in combination with the $\mathrm{N}$ cytoplasm: Beadle (1932) has described 15 different ms mutants in cytoplasm $\mathrm{N}$ background, but these are considered to represent genic male sterility in maize breeding. However, as long as all these ms mutants have not been tested for their expression in the three CMS backgrounds, the possibility exists that the $\mathrm{N}$ cytoplasm actually represents a fourth CMS type in maize (see Engelke and Tatlioglu (2000) for a well-tested example of genic ms).

The number of CMS types can easily be underestimated. First, the number of field sources tested sets an upper limit to how many of the CMS types present can be discovered (Frank, 1989). Testing a field source against a single known CMS type in this study required on average raising 750 progeny, and the number of comparisons rises linearly with the number of CMS types known. Second, when a reciprocal test does not yield a significant segregation difference, this can be due to symmetry in restorer genotypic composition between the two parents involved, even though they have different CMS types. The power of the analysis is therefore strongly dependent on the number of test plants used with different restorer genetic backgrounds. A good example of this in the present study comes from the reexamination of plant kh120 previously determined by Koelewijn and Van Damme (1995a) as CMS1. Adding crosses with a number of descendants of the original test plant clearly revealed kh120 to be different from CMS1 (Table 4).

All new CMS sources in this study showed segregation ratios below 1:3, suggesting more than one restorer locus to be involved in male fertility restoration in each CMS type, which is in line with earlier studies in $P$. coronopus (Koelewijn and Van Damme, 1995b) and in other gynodioecious species (Van Damme, 1983; Charlesworth and Laporte, 1998). The occurrence of multiple restorer genes per CMS type, either recessive or dominant or a combination of both, observed in a number of wellstudied species (references above), is ill understood. Models on nuclear-cytoplasmic male sterility typically assume a single locus to restore the male sterility effect of CMS (Gouyon et al, 1991; Maurice et al, 1994; Schultz, 1994; McCauley and Taylor, 1997; Bailey et al, 2003). It is hard to see what selective advantage would allow a restorer allele of a second locus to invade into a population where already a restorer locus is close to fixation.

Of all theoretical studies on the evolutionary dynamics of gynodioecy, only Frank (1989) has explicitly modeled a situation with a large number of CMS systems with more than one restorer locus per CMS type. In a stochastic model with a potential of 16 cytoplasmic types, Frank found that more than three CMS types were present in the population for a considerable fraction of time. However, his restorer gene model assumed the different CMS types to have restorer genes in common. There is currently no evidence for this specific type of nuclearcytoplasmic interaction in gynodioecious species, and it remains unclear how Frank's specific genetic 
assumptions have influenced the conditions for maintenance of multiple CMS systems.

The mechanism behind CMS is generally considered to be occurrence of chimeric genes in the mt-DNA that are expressed in the cell, somehow leading to male sterility at the plant level (Saumitou-Laprade et al, 1994; Schnable and Wise, 1998). These chimeric genes are thought to arise by the so-called rearrangements involving inverted repeats. Interestingly, an unusually high mt-DNA sequence divergence in the genus Plantago has been reported by Palmer et al (2000), suggesting a higher than average point mutation frequency in this genus. It is however unclear whether this peculiar phenomenon contributes to the multiple CMS-restorer systems in both $P$. lanceolata (De Haan et al, 1997) and P. coronopus.

In conclusion, our finding that $P$. coronopus has at least four co-occurring CMS-restorer gene systems raises the questions how these systems are distributed in the field, to what extent they co-occur within populations and how these aspects do affect the evolutionary dynamics of the gynodioecious sex system.

\section{Acknowledgements}

We thank Joop van Heeswijk for collecting the male sterile plants from Achill Island in Ireland, Gerard Koorevaar for making available plants from cross G140, and Arjen Biere for critical comments on earlier versions of the manuscript.

\section{References}

Bailey MF, Delph LF, Lively CA (2003). Modeling gynodioecy: novel scenarios for maintaining polymorphism. Am Nat 161: 762-776.

Beadle GW (1932). Genes in maize for pollen sterility. Genetics 17: 413-431.

Beckett JB (1971). Classification of male sterile cytoplasms in maize (Zea mays L.). Crop Sci 11: 724-727.

Belhassen E, Atlan A, Couvet D, Gouyon PH, Quetier F (1993). Mitochondrial genome of Thymus-vulgaris L. (Labiate) is highly polymorphic between and among natural-populations. Heredity 71: 462-472.

Belhassen E, Dommee B, Atlan A, Gouyon PH, Pomente D, Assouad MW et al (1991). Complex determination of malesterility in Thymus-vulgaris L. - genetic and molecular analysis. Theor Appl Genet 82: 137-143.

Charlesworth D, Laporte V (1998). The male-sterility polymorphism of Silene vulgaris: analysis of genetic data from two populations and comparison with Thymus vulgaris. Genetics 150: $1267-1282$.

Cuguen J, Wattier R, Saumitou-Laprade P, Forcioli D, Morchen M, Vandijk $H$ et al (1994). Gynodioecy and mitochondrial DNA polymorphism in natural populations of Beta vulgaris sp maritima. Genet Sel Evol 26: S87-S101.

De Haan AA, Mateman AC, Van Dijk PK, Van Damme JMM (1997). New CMS types in Plantago lanceolata and their relatedness. Theor Appl Genet 94: 539-548.

Duvick DN (1965). Cytoplasmic pollen sterility in corn. Adv Genet 13: 2-56.
Engelke T, Tatlioglu T (2000). Genetic analyses supported by molecular methods provide evidence of a new genic (st1) and a new cytoplasmic (st2) male sterility in Allium schoenoprasum L. Theor Appl Genet 101: 478-486.

Fauron CMR, Casper M (1994). A second type of normal maize mitochondrial genome: an evolutionary link. Genetics 137: 875-882.

Fauron CMR, Moore B, Casper M (1995). Maize as a model of higher plant mitochondrial genome plasticity. Plant Sci 112: 11-32.

Frank SA (1989). The evolutionary dynamics of cytoplasmic male sterility. Am Nat 133: 345-376.

Gouyon PH, Vichot F, Van Damme JMM (1991). Nuclearcytoplasmic male-sterility - single-point equilibria versus limit-cycles. Am Nat 137: 498-514.

Kheyr-Pour A (1981). Wide nucleo-cytoplasmic polymorphism for male sterility in Origanum vulgare L. J Hered 72: 45-52.

Koelewijn HP, Van Damme JMM (1995a). Genetics of malesterility in gynodioecious Plantago-coronopus. 1. Cytoplasmic variation. Genetics 139: 1749-1758.

Koelewijn HP, Van Damme JMM (1995b). Genetics of malesterility in gynodioecious Plantago-coronopus. 2. Nuclear genetic-variation. Genetics 139: 1759-1775.

Laporte V, Merdinoglu D, Saumitou-Laprade P, Butterlin G, Vernet P, Cuguen J (1998). Identification and mapping of RAPD and RFLP markers linked to a fertility restorer gene for a new source of cytoplasmic male sterility in Beta vulgaris ssp. maritima. Theor Appl Genet 96: 989-996.

Lewis D (1941). Male sterility in natural populations of hermaphrodite plants. New Phytol 40: 56-63.

Lewis D, Crowe LK (1956). The genetics and evolution of gynodioecy. Evolution 10: 115-125.

Maurice S, Belhassen E, Couvet D, Gouyon PH (1994). Evolution of dioecy - can nuclear-cytoplasmic interactions select for maleness. Heredity 73: 346-354.

McCauley DE, Taylor DR (1997). Local population structure and sex ratio: evolution in gynodioecious plants. Am Nat 150: 406-419.

Palmer JD, Adams KL, Cho YR, Parkinson CL, Qiu YL, Song KM (2000). Dynamic evolution of plant mitochondrial genomes: mobile genes and introns and highly variable mutation rates. Proc Natl Acad Sci USA 97: 6960-6966.

Ronfort J, Saumitou-Laprade P, Cuguen J, Couvet D (1995). Mitochondrial-DNA diversity and male-sterility in natural populations of Daucus-carota ssp carota. Theor Appl Genet 91: 150-159.

Saumitou-Laprade P, Cuguen J, Vernet P (1994). Cytoplasmic male-sterility in plants - molecular evidence and the nucleocytoplasmic conflict. Trends Ecol Evol 9: 431-435.

Schnable PS, Wise RP (1998). The molecular basis of cytoplasmic male sterility and fertility restoration. Trends Plant Sci 3: 175-180.

Schultz ST (1994). Nucleocytoplasmic male-sterility and alternative routes to dioecy. Evolution 48: 1933-1945.

Snedecor GW, Cochran WG (1977). Statistical Methods. Iowa State University Press: Ames.

Ullstup AJ (1972). The impacts of the southern corn leaf blight epidemic of 1970-1971. Ann Rev Phytopathol 10: 37-50.

Van Damme JMM (1983). Gynodioecy in Plantago lanceolata L. II. Inheritance of three male sterility types. Heredity 50: 253-273.

Van Damme JMM, Van Delden W (1982). Gynodioecy in Plantago lanceolata L. I. Polymorphism for plasmon type. Heredity 49: 303-318. 